\title{
Yes! NLP-based FL-ITS will be Important
}

\author{
Henry Hamburger \\ Department of Computer Science \\ George Mason University \\ Fairfax VA 22030 USA \\ henryh@cs.gmu.edu
}

In this note, a straw man is destroyed, optimism is expressed, an existing system is sketched, and some issues are laid out.

\begin{abstract}
A Direct Approach.
It is useful to consider a straightforward argument for according NLP a central role in CALL. I will call it SCW, since it is simple, clear and wrong. It runs like this: intelligent tutoring systems (ITS) with simulated problem environments are potentially excellent for learning; a key module of an ITS is a computational model of expertise in the domain; NLP systems are such models in the domain of human languages; so let's use NL.P knowledge bases as the expert module of a foreign language ITS.
\end{abstract}

\section{But Language is Different.}

But language use is different from most ITS domains, in a way that invalidates SCW. In a typical domain a student shows progress by success in explicit stepwise reasoning to a solution. Successful language use, in contrast both to other ITS domains and to the ability to state grammatical rules, need not demand articulating the stepwise reasoning about sentence construction, but it does require the capacity to arrive quickly at a result (either a sentence that expresses one's current thought or the meaning of someone else's sentence), and moreover to do so while thinking about something else, namely the substance of the conversation. Such a capacity may well imply a grammar in the brain but not awareness and articulation of it.

\section{Expertise and ITS.}

Even for explicit teaching of grammatical, lexical or other knowledge of a particular language, SCW is flawed. Experience teaches that a performanceoriented representation of domain knowledge may be unsuitable for direct use in an ITS; see Clancey (1987) and in the world of language specifically, see Chanier et al. (1992). For a rule to be learned or even to be useful in explanation, it should be congruent with learners' cognition and expressed in a way that is meaningful to them. Anyone familiar with NIP knowledge bases could hardly think seriously about promoting their direct use in explanations to learners.

Stay the Course.

The failure of SCW certainly does not mean we should all go home. On the contrary, I strongly believe that valuable ITSs will be built and that they will use NLP, or I would not spend so much of my time on one. It does, however, suggest that it will take significant work, in design as well as implementation, to create an NLP-driven ITS for language that engages students and helps then learn efficiently.

\section{Let Many Flowers Bloom.}

Proclaiming a future for NLP in ITS is not to deny benefits from other kinds of devices or the continuing importance of human teachers, tutors and other conversational partners. Indeed NI.P itself may have variegated ways of contributing to the mix of learning resources, by systems that use different modules of NLP and that aim to foster various kinds of language knowledge in the student. Automated aid has been undertaken for parts of languages all the way from spelling, pronunciation and morphology, through syntax and semantics, to discourse and cultural knowledge. For the most part, the NLP-based work has been at the low end, rarely going above syntax.

\section{Two-Medium Conversation.}

A sketch of our own (Hamburger, 1995) twomedium conversational system will give a concrete sense of one approach to NLP-intensive CALL. FLUENT-2 is a language learning and tutoring system, helping students learn and letting teachers influence what is learned. It has features of both intelligent tutoring systems and microworld learning environments. Using a pedagogical 
strategy of situational immersion, the system engages the student in meaningful multi-media communicative acts in graphically depicted real world situations.

The student interacts with the system by direct use of the target language. The system uses Felshin's (1993) multilingual NLP system, which can expose students to a wide variety of linguistic forms. The intent is that new words, phrases and grammatical usage will become comprehensible through meaningful exposure and use. The system makes this possible by tightly coupling the language to graphical acts and system generated animations within a realistic ongoing situation. Actions available to both the student and the system include selecting and moving objects and making human figures walk, turn, point, grasp and release objects, and so on.

The teacher interacts with the system through graphical (GUI) tools that facilitate the designing of exercises and the construction of appropriate microworlds. These tools let a teacher construct exercises that invoke specific linguistic concepts in the target language without having to deal directly with the NLP system. One parameter of an exercise can be a plan for a goal in a situation, a capacity that makes exercises portable across microworlds. Real language teachers have given design advice and now use the system.

\section{Three Issues.}

Fidelity and interactiveness are key issues for ITS. Extensibility has been a key issue in NLP. All three will be important for NLP-based CALL. I'll subdivide each, mention some interplay among them and comment on Fluent- 2 in light of them.

Fidelity is the accuracy of a presentation. For example the visual fidelity of a photo or video exceeds that of simple graphics or animation. A technical drawing may have good conceptual fidelity if it connects related concepts. Two aspects of fidelity that may rightly concern language educators are cultural authenticity and the situational continuity of a conversation.

Interactiveness of an ITS can include its immediate responsiveness by faithfully updating the visible situation after a student makes some kind of move as well as longer-range responsiveness to an individual student, based on a model that it builds of that student's knowledge. In addition, the student may be offered control over parameters of the systems behavior, including subject matter, difficulty and style. We have used this last approach, in response to arguments made by Self (1988).

A system can be designed to be extendable within a language. It takes extra effort to make it possible for a teacher to do so, as opposed to a programmer. Portability across languages is familiar to NLP researchers, and, as noted above, portability can also refer to moving exercise types into new situations. Schoelles and Hamburger (1996) show how this capacity lets one present a language concept in one situation and test it in another.

There are tradeoffs here. As an example, Murray's (1995) video system achieves exceptionally fine cultural fidelity, but for that reason little of it is language portable. Interactiveness also is difficult to achieve with video, since there is a finite amount of video material produced in advance. Our generative, recombinative animation approach does not encounter this constraint.

\section{In the End.}

It will not be easy, but I hope and believe that before too long there will be a variety of exciting, effective NLP-based CALL systems.

\section{References}

Chanier, T., Pengelly, M., Twidale, M. and Self, J. (1992) Conceptual modelling in error analysis in computer-assisted language learning systems. In Swartz, M.L. and Yazdani, M. (Eds.) Intelligent Tutoring Systems for Foreign Language Learning. Berlin: Springer-Verlag

Clancey, W. (1987) Knowledge-Based Tutoring: The GUIDON Program. Cambridge, MA, USA: MIT Press.

Felshin, S. (1993) A Guide to the Athena Language Learning Project Natural Language Processing Systems. MIT.

Hamburger, H. (1995) Tutorial tools for language learning by two-medium dialogue. In Holland, V.M., Kaplan, J.D. and Sams, M.R. (Eds.) Intelligent Language Tutors. Mahwah, NI, USA: Erlbaum.

Murray, J. (1995) Lessons learned from the Athena Language Learning Project. In Holland, V.M., Kaplan, J.D. and Sams, M.R. (Eds.) Intelligent Language Tutors. Mahwah, NJ, USA: Erlbaum. 
Schoelles, M. and Hamburger, H. (1996) Teacherusable exercise design tools. Montreal: Proceedings of ITS-96.

Self, J.A. (1988) Bypassing the intractable problem of student modelling. Montreal: ITS- 88 . 